\title{
INCONGRUÊNCIA DO COTOVELO: revisão
}

\author{
Elbowinonguity: review
}

\author{
Thaís Andrade Costa ${ }^{a}$, Renata Moris Domenico O liveirab, \\ Sam Goldy Shoyama 0 da $^{c}$, Julia Maria Matera ${ }^{d}$
}

\footnotetext{
a MédicaV eterinária, Mestranda em Cirurgia(FMVZ/ USP), São Paulo, SP - Brasil, e-mail: thaiscosta@ yahoo.com.br

b Médica V eterinária, Mestranda em Cirurgia(FMVZ/US), São Paulo, SP - Brasil, e-mail: renatamd@ hotmail.com

c Médico Veterinário, Mestrando em Cirurgia(FMVZ/ USP), São Paulo, SP - Brasil, e-mail: sgoda@ superig.com.br

d MédicaV eterinária, ProfessoraTitulardo D epartamento deCirurgia(FMVZ/ USP), São Paulo, SP - Brasil, e-mail:materajm@ usp.br
}

\section{Resumo}

A articulação do cotovelo é sede das principais afecções responsáveis pela claudicação em membros torácicos em cães. Trata-se de uma articulação complexa composta pela porção distal do úmero e porções proximais do rádio e ulna. A incongruência do cotovelo é caracterizada por uma má formação dos componentes ósseos dessa articulação resultando em desnivelamento entre rádio e ulna ou tróclea mal formada. Afecções como não união do processo ancôneo, osteocondrite dissecante do côndilo umeral medial e fragmentação do processo coronoide medial da ulna são agrupadas com a denominação de displasias do cotovelo. D iscute-se, atualmente, a inclusão da incongruência do cotovelo como uma quarta afecção da displasia do cotovelo e o seu papel como fator predisponente ao desenvolvimento das demais afecções. Há diversos estudos sobre a sensibilidade e a acurácia dos diferentes métodos diagnósticos existentes, sendo a to mografia computadorizada o melhor método atualmente em uso. 0 procedimento cirúrgico mais utilizado e amplamente aceito é a osteotomia proximal e oblíqua da ulna com aplicação de pino intramedular para estabilização da porção proximal da ulna, cuja técnica vem apresentando bons resultados quanto à presença de dor e retorno à função do membro no período pós-operatório. 0 diagnóstico e o tratamento precoce, e em animais jovens, geralmente possuem um melhor prognóstico. Há um aumento constante das pesquisas relacionadas à incongruência do cotovelo, envolvendo a sua fisiopatogenia, formas de diagnóstico e tratamento, na tentativa de um maior entendimento dessa afecção, por se tratar de uma enfermidade diagnosticada há pouco mais de duas décadas.

Palavras-chave: Cotovelo; Incongruência; D isplasia; Cães. 


\begin{abstract}
The disorders that affect the elbow joint are the main responsible for lameness in thoracic limb displayed by dogs. It is a complex, accurately matching joint formed by the distal humerus and the proximal radius and ulna. The elbow incongruity is characterized by armuntidiness of its joint surfaces resulting in a step between the radius and the ulna or an elliptical trochlea. Over the last two decades, elbow incongruity has become considered as a cause of elbow dysplasia, however it has been considered that it should be included as a fourth disorder. It has been also suggest that among the disposable diagnostic methods the computed tomography is a more sensitive and specific one. The proximal oblique ulnar osteotomy with intra-medullary pin is the most frequently surgical procedure performed for the treatment of incongruent elbows showing good results at the postoperative period. An early diagnosis ant treatment of this disorder results in a better prognosis. There are an increasing number of studies involving aetiology, diagnostic methods and surgical treatment of elbow incongruity.
\end{abstract}

Keywords: Elbow; Incongruity; Dysplasia; Dogs.

\title{
INTRODUÇÃO
}

O cotovelo é uma articulação complexa composta pelo úmero distal, rádio e ulna proximais. A incongruência do cotovelo (IC) é caracterizada pelo mau alinhamento de um desses três ossos dentro da articulação (HULSE; JO HNSO N, 2002).

Incongruência do cotovelo foi descrita primeiramente em 1982 através de radiografias de cães da raça Bernese Moutain D og acometidos por fragmentação do processo coronoide medial da ulna (BLO ND et al., 2005). D urante as últimas duas décadas a IC vem sendo considerada uma causa da displasia de cotovelo, a qual é constituída de três processos patológicos que resultam em fragmentos soltos dentro da articulação levando à dor e à artrose. O s três processos patológicos são: não união do processo ancôneo (NUPA), fragmentação do processo coronoide (FPC) e osteocondrite dissecante da porção medial do côndilo do úmero (OCD) (MASO N; SCHULZ; SAMII, 2002; PRESTO N; SCHULZ; KASS, 2000). Em 1986, Wind sugeriu que a incongruência da articulação do cotovelo é um denominador comum nas três desordens mencionadas anteriormente.

De acordo com o grupo internacional de trabalho com cotovelo (International Elbow Working Group - IEWG), a incongruência é a quarta disfunção do cotovelo, devendo ser agrupada dentro da denominada displasia do cotovelo (SAMOY et al., 2006).

A IC ocorre, mais frequentemente, em cães jovens de raças grandes. D entre essas características as fêmeas parecem ser menos acometidas do que os machos de crescimento rápido. D oenças do cotovelo são a causa comum de claudicação de membros torácicos em cães (WIND , 1986).

\section{Anatomia da articulação do cotovelo}

O cotovelo é uma articulação composta por três superfícies articulares, úmero, rádio e ulna, que combinam em uma única cápsula, formando uma articulação tipo dobradiça (gínglimo). Suas estruturas articulares se sobrepõem, dificultando a visibilização das suas estruturas internas (DYCE et al., 2004).

A porção distal do úmero é o côndilo, que articula com a incisura troclear, também chamada de incisura troclear da ulna. A região medial do úmero é a tróclea do úmero, ou côndilo umeral medial. A porção lateral do côndilo do úmero é chamada de capítulo, responsável por mais de $80 \%$ do suporte do peso, articulando com a cabeça do rádio. A articulação úmero-ulnar é formada pela estreita articulação do côndilo medial (tróclea) com o processo coronoide medial da ulna, a qual é parte da incisura troclear ou semilunar da ulna proximal e é responsável por uma menor porcentagem da sustentação do peso na 
articulação (BOULAY, 1998). No úmero há um forame supra-articular, onde se encaixa o processo ancôneo da ulna, durante o movimento de extensão. Na porção medial do rádio ocorre o processo coronoide medial e na ulna o processo coronoide lateral. Os ligamentos mais resistentes são ligamentos colaterais medial e lateral, um arranjo previsível em uma articulação do tipo dobradiça (DYCE et al., 2004). $O$ ligamento anular corre transversalmente à cabeça do rádio mantendo a articulação rádio-ulnar. Esse ligamento também passa por ambos os lados do processo coronoide, lateral e medial, abaixo dos ligamentos colaterais correspondentes medial e lateral (BO ULAY , 1998). O s movimentos mais importantes são os de extensão e flexão, havendo pequenos movimentos de pronação e supinação, como resultado da articulação entre o rádio e a ulna, através do ligamento interósseo (HORNOFF et al., 2000).

No cotovelo incongruente, a superfície articular não está bem alinhada. Um crescimento coordenado da metáfise e da placa de crescimento são necessários durante o desenvolvimento da articulação do cotovelo (SAMOY et al., 2006). O cotovelo imaturo possui seis placas de crescimento. 0 úmero distal tem três centros de ossificação secundários; os côndilos medial e lateral, os quais se fundem, e 0 epicôndilo medial separado. A ulna proximal possui dois centros de ossificação: a apófise do olécrano e o processo ancôneo. A ossificação do processo coronoide e a fusão do processo ancôneo são completadas aproximadamente na $20^{\mathrm{a}}$ até a $22^{\mathrm{a}}$ semana de idade. 0 rádio possui uma placa de crescimento distal e outra proximal. $\mathrm{O}$ crescimento longitudinal da ulna é gerado apenas pela placa de crescimento distal e irá originar 0 crescimento longitudinal do antebraço pela combinação do crescimento das placas proximal e distal do rádio. A placa de crescimento proximal da ulna e a cartilagem articular da placa de crescimento da ulna proximal contribui apenas para o formato da ulna proximal e do olécrano (BOULAY, 1998).

\section{Definição e etiopatogenia da incongruência}

A incongruência articular tem sido definida como má-formação e desalinhamento da articulação do cotovelo. As alterações observadas incluem o aumento do espaço articular entre o úmero e a ulna e entre 0 úmero e o rádio, a formação de "degrau" ósseo entre o processo coronoide e a cabeça do rádio, a incongruência troclear e a esclerose subcondral (SCHWARZ, 2000).

A incongruência era definida apenas como desenvolvimento anormal da incisura troclear da ulna, resultando em uma superfície articular levemente elíptica com um arco de curvatura pequeno em comparação com a tróclea umeral. Isso poderia criar uma articulação com maior ponto de contato nas áreas dos processos ancôneo e coronoide, mas sem um ponto de contato entre a incisura troclear e a tróclea umeral. Isso é explicado porque a taxa de crescimento da parte proximal da ulna deve ser mais rápida para que acompanhe a taxa de crescimento do côndilo umeral, porém, se por alguma razão este crescimento da ulna é retardado, o tamanho da incisura troclear fica pequeno em relação ao côndilo umeral (WIND , 1986). O crescimento assincrônico entre a tróclea umeral e a incisura troclear da ulna, resulta em uma incongruência e um crescimento longitudinal assincrônico do rádio e da ulna, resultando em um degrau intra-articular no cotovelo (PRESTON et al., 2001).

Preston et al. (2000) realizaram um estudo da determinação das áreas de contato em articulações úmero-rádio-ulnar normais de dez membros de cinco cadáveres de cães e observaram a existência de uma incongruência côncava fisiológica entre a superfície de sustentação do peso do úmero e da ulna. Isso ocorre por uma diferença de formato, resultando em um contato articular periférico e aumento do espaço intra-articular. A incongruência geométrica de articulações normais serve para otimizar a distribuição do estresse durante a carga, produzindo uma transmissão bicêntrica de forças de carga baixa. Esses autores sugeriram que a incongruência fisiológica produz uma estimulação intermitente do tecido cartilaginoso, e tem efeitos benéficos no metabolismo, nutrição e lubrificação da cartilagem articular durante a carga cíclica. 0 crescimento assincrônico do rádio e da ulna, criando um degrau no aspecto medial da articulação rádio-ulnar, deve contribuir para uma distribuição de estresse anormal e uma sobrecarga mecânica potencial do processo coronoide medial.

A tualmente, divide-se a incongruência patológica em duas formas. A primeira é a descrita anteriormente por Wind (1986) causada pela forma elíptica da incisura troclear com diâmetro pequeno em comparação ao côndilo umeral, o que aumenta a pressão sobre o processo ancôneo e o processo

Rev. Acad., Ciênc. Agrár. Ambient., Curitiba, v. 6, n. 3, p. 413-423, jul./ set. 2008 
coronoide medial. A outra é um crescimento inadequado entre o rádio e a ulna (rádio curto/ ulna curta) que, em casos mais graves, causarão desordens como valgus, ou varus e de crescimento em cães de diferentes raças. Em casos de crescimento discreto do rádio ou de ulna, as mudanças externas são menos pronunciadas. Esse crescimento inadequado do rádio ou da ulna, pode ser resultante de causas congênitas e hereditárias nutricionais ou traumáticas (SJÖ STRÖ M; KASSTRO N; KALLBERG , 1995).

A elevação da pressão sobre a superfície articular durante o desenvolvimento pode causar um aumento local da pressão transarticular em alguns pontos e subsequente fissura, fragmentação do osso e destruição da cartilagem articular. A excessiva pressão local tem sido associada com morte dos condrócitos, danos à matriz cartilaginosa, e início de osteoartrite (PRESTON et al., 2000). A fragmentação do processo coronoide medial (FPCM) é mais comumente observada associada à incongruência articular e a alterações secundárias à incongruência (HORNOF et al., 2000).

A displasia do cotovelo (D C) acomete cães machos e fêmeas de faixa etária compreendida entre cinco e oito meses de idade; é considerada uma das causas mais frequentes de doença articular degenerativa em cotovelos de cães. Schwarz (2000) acredita que a IC é um importante fator para 0 aparecimento da DC, assim como as outras causas, como a FPCM, NUPA e a OCD. Todavia, outros autores contestam esta afirmação.

A incongruência articular (IA) e a FPCM são os primeiros sinais clínicos encontrados em um estudo de displasia de cotovelo em 520 pastores alemães. A IA foi observada em 85\% dos casos dos animais com displasia. A combinação das lesões em animais displásicos foi muito comum. Foi observado que a IA está sempre presente quando há mais de uma lesão nestes animais, corroborando com a hipótese de que a incongruência articular é uma das afecções de displasia do cotovelo (REMY et al., 2004).

A consequência biomecânica de uma incongruência rádio-ulnar por degrau é desconhecida. É possível que o evento inicial da patogênese da D C seja um crescimento longitudinal assincrônico do rádio e da ulna, resultando em um degrau intra-articular. Isso altera 0 contato articular e a transmissão de carga. $O$ aumento da pressão na articulação incongruente leva à displasia (PRE STON et al., 2001).

Nos casos de incongruência causada por uma ulna curta, a incisura troclear é empurrada em sentido distal e o processo ancôneo comprime a tróclea umeral (HULSE; JOHNSO N, 2002), causando um aumento da pressão no processo ancôneo, o qual evolui para a fragmentação ou não união devido a separação do centro de ossificação, uma condição que tem sido descrita em inúmeras raças (PUCCIO et al., 2003). Wind (1986) afirma que esse retardo de crescimento pode ser compensado por um estágio posterior, o qual explica a presença de fragmentos sem um sinal claro de incongruência.

Já nos casos de incongruência causada por rádio curto, a cabeça radial é impelida em sentido distal e não se articula com o capítulo umeral. A tróclea do úmero então se situa diretamente no processo coronoide da ulna, que transmite to da a força de sustentação do peso (HULSE; JO HNSO N, 2002). Há um aumento da pressão na porção medial do côndilo umeral e do processo coronoide medial, o que leva à claudicação e fragmentação desse processo coronoide (SAMOY et al., 2006).

Esse aumento da pressão sobre o processo coronoide foi observado por Preston et al. (2001) em um estudo in vitro com 17 articulações incongruentes, em que determinou as áreas de contato, tendo observado que o resultado do encurtamento do rádio foi o de redução do tamanho das áreas de contato radiais, uma redução ou perda do contato da área ulnar proximal, alargamento do aspecto distal da incisura ulnar troclear da área de contato na região do processo coronoide lateral, e migração da área de contato do coronoide para a projeção lateral do processo coronoide medial. A redução da área de contato combinada rádio-ulnar, sugere que o nível da superfície incongruente, atribuída ao rádio mais curto pode aumentar a pressão local na cartilagem articular e no osso subcondral subjacente. 0 degrau articular incongruente pode concentrar as forças fisiológicas na margem elevada medial do processo coronoide, resultando em uma fragmentação osteocondral. Esses achados sustentam as causas mecânicas de lesão no coronoide encontradas em casos espontâneos de displasia articular do cotovelo. O tipo e a localização dos fragmentos osteocondrais encontrados em casos espontâneos de displasia do cotovelo sugerem que o degrau de incongruência pode contribuir para a patogênese dessa doença. 
Em contradição com Preston et al. (2001), um estudo de tomografia computadorizada não demonstrou aumento da pressão articular afetada pela FPCM, quando comparada com articulações normais (FUJITA et al., 2003). Preston et al. (2001) realizando osteotomia ulnar para corrigir a deformidade articular, evitaram o desenvolvimento da FPCM e da NUPA, o que reafirma a hipótese da incongruência articular do cotovelo ser uma das causas de displasia.

A IC, especificamente a incisura troclear da ulna, é um fator comum de cães com FPCM, OCD e NUPA. A curvatura reduzida pode resultar em pontos de contato entre o côndilo umeral e o processo ancôneo e o processo coronoide medial. O grau de incongruência pode afetar mais ou menos essas áreas de contato, predispondo a fissura ou fragmentação do processo coronoide medial. Se a taxa de crescimento for capaz de prender a tróclea umeral, isso pode explicar o porquê de algumas fragmentações do coronoide cicatrizarem (BO ULAY , 1998). O utros autores contestam essa correlação de uma incisura troclear ulnar elíptica com o desenvolvimento de fragmentação do processo coronoide medial e de não união do processo ancôneo, pois relatam ser rara a ocorrência das duas últimas enfermidades simultaneamente na mesma articulação (KIRBERG ER; FOURIE, 1998; REMY et al., 2004).

\section{Sinais clínicos}

A incongruência articular é mais comum em cães imaturos, pois suas fises ainda estão abertas, podendo haver um fechamento precoce delas, após trauma ou por alterações de crescimento. O utros podem sofrer por um crescimento assincrônico da articulação. 0 animal pode ser apresentado no início do problema, ou quando idoso, e com doença articular degenerativa secundária estabelecida (HULSE; JOHNSON, 2002).

Os animais afetados quase sempre possuem histórico de claudicação intermitente, que pode vir acompanhada por trauma anterior ou não. E ssa claudicação pode ser de graus variados e não se sabe a real associação dessa condição com a claudicação, pois geralmente outras enfermidades vêm associadas com a NUPA, a FPCM e a O CD , por exemplo, não sendo possível distinguir a origem dessa claudicação (SAMOY et al., 2006).

Os sinais clínicos mais consistentes encontrados são: a dor à palpação do processo coronoide medial e da manipulação da articulação, a efusão articular, e a restrição de movimentação articular (PUCCIO et al., 2003), sendo também citado a crepitação e a atrofia muscular. A deformidade macroscópica do membro pode estar presente, dependendo da placa fiseal afetada e a relação da lesão com o crescimento do animal (HULSE; JOHNSO N, 2002). No geral, os animais mais novos têm um maior risco de desenvolvimento de fragmentos perdidos, porque a ossificação ainda não está completa e seus ossos são mais frágeis e menos densos (SAMOY et al., 2006).

\section{Diagnóstico}

O diagnóstico de incongruência do cotovelo pode ser difícil (GEMMILL et al., 2006). A radiografia é a técnica de eleição para diagnóstico de doenças do cotovelo em cães, já que permite 0 diagnóstico da NUPA, O CD e raramente de FPC (SAMOY et al., 2006). 0 diagnóstico radiográfico de osteoartrose tem sido estudado intensivamente e técnicas radiográficas que melhorem a sensibilidade da detecção de fragmentação do coronoide têm sido relatadas (HO LSWORTH et al., 2005). Mudanças degenerativas da articulação úmero-rádio-ulnar geralmente não são detectadas radiograficamente antes dos 7 a 8 meses de idade (OLSSO N, 1983).

Três projeções são recomendadas para avaliação da articulação úmero-rádio-ulnar: a médiolateral, em flexão e extensão e uma projeção oblíqua crâniomedial-craniolateral (MASO N et al., 2002). A congruência do cotovelo é mais bem avaliada na projeção médio-lateral com articulação flexionada a $90^{\circ}$ e com a direção dos raios centrada na articulação (MURPHY et al., 1998). O cotovelo normal, nessa projeção, é caracterizado por um espaço articular simétrico entre o úmero, o rádio e a ulna, criando um arco semicircular contínuo perfeito. A projeção crânio-caudal não permite o diagnóstico da incongruência 
articular. Incongruência do cotovelo pode ser notada por radiografia, mas defeitos mínimos de degrau entre o rádio e a ulna não são percebidos por esta modalidade diagnóstica (BLO ND et al., 2005).

A avaliação radiográfica da incongruência não é simples. É difícil visualizar uma estrutura tridimensional em uma projeção bidimensional. A radiografia não é sensível suficientemente para avaliar incongruência por causa da influência de sobreposição de imagens e da necessidade de posicionamento perfeito da articulação (MASO N et al., 2002).

Blond et al. (2005) avaliaram in vitro a sensibilidade e a especificidade da radiografia na detecção de incongruência do cotovelo em cães. Foram avaliados cinco cotovelos esquerdos de cadáveres caninos com peso superior a 20 quilos. Foram criados mecanicamente defeitos de 3, 2 e $1 \mathrm{~mm}$ no rádio distal desses membros. Foram realizadas projeções médio-laterais em dois ângulos diferentes de $90^{\circ}$ e $135^{\circ}$ de flexão, e 10 posições do feixe de R-X diferentes. Os resultados mostraram boa sensibilidade para detecção de incongruência em defeitos de até $2 \mathrm{~mm}$, na posição de flexão a 90 (100\%) e 85\% na posição de flexão a 135․ Em defeitos de $1 \mathrm{~mm}$ a sensibilidade foi baixa, $60 \%$, e simulando 0 apoio do membro diminuiu ainda mais para 50\%. A especificidade foi diferente entre os 3 avaliadores e variou de 70 a $90 \%$. A melhor especificidade foi obtida pelo melhor radiologista sugerindo que a experiência pode influenciar a detecção de cotovelos normais. Os autores concluem que a radiografia é um teste sensível e específico para detectar incongruência de grau leve a moderado (2 mm ou mais) se 0 cotovelo estiver flexionado a 90 graus e o feixe de raios centrado na articulação. D etecção radiográfica e quantificação de incongruência rádio-ulnar são mais contestáveis. Publicações reportando achados radiográficos de IC baseiam-se somente em técnicas de mensuração subjetivas; técnicas de mensuração objetivas não são descritas (HORNOF et al, 2000; MURPHY et al., 1998). Radiografia simples não é capaz de detectar incongruência sutil (MURPHY et al., 1998; MASO N et al., 2002).

Tomografia computadorizada (TC) é a melhor técnica para avaliação e mensuração da incongruência do cotovelo (REICHLE et al., 2000; SAMOY et al., 2006), pois produz resolução excelente de estruturas ósseas, permitindo uma avaliação completa da articulação do cotovelo, sem a presença da sobreposição de imagens que ocorre na radiografia simples (G EMMILL, 2006; REICHLE et al., 2000; ROVESTI et al., 2002). As reconstruções sagital e dorsal são as melhores para diagnosticar a incongruência do cotovelo (REICHLE et al., 2000). Segundo Holsworth et al. (2005) parece não haver perda de acurácia na avaliação de congruência úmero-rádio-ulnar em imagens reconstruídas no plano transverso, quando comparadas com imagens reconstruídas nos planos sagital e dorsal.

Holsworth et al. (2005) estudaram a acurácia da tomografia computadorizada na avaliação de incongruência rádio-ulnar in vitro, através da análise de imagens obtidas de 8 membros torácicos de cadáveres caninos. Foram criados mecanicamente defeitos no comprimento do rádio de 1, 2, e $4 \mathrm{~mm}$, e imagens tomográficas reconstruídas nos planos sagital, dorsal e dorsal oblíquo foram realizadas. Medidas obtidas pelo computador e medidas obtidas através da mensuração direta das peças anatômicas foram comparadas. Os autores concluem que: a medida da congruência é mais bem avaliada no plano dorsal oblíquo, na porção média do coronoide; a aplicação de protocolos de avaliação da incongruência do cotovelo melhora a aplicação da técnica cirúrgica. A única limitação é que a medida da congruência é feita sobre o osso subcondral e não sobre a cartilagem.

G emmill et al. (2006) compararam imagens tomográficas reconstruídas nos planos sagital e dorsal de 8 cotovelos de cadáveres caninos e entre 2 observadores diferentes. Foram obtidas medidas do espaço articular com variações mínimas entre as medidas tomográficas e as medidas das peças anatômica e entre os 2 observadores, ressaltando, portanto, a importância da TC no diagnóstico de incongruência de cães com DC.

Kramer et al. (2006) avaliaram a incongruência articular rádio-ulnar in vitro através da tomografia computadorizada com diagnóstico de "doença do compartimento medial" (FPC, OCD, desgaste da cartilagem do processo coronoide medial e da tróclea da ulna). Foram comparadas imagens de animais com doença e cães saudáveis. Os resultados mostraram ausência de significância estatística para incongruência rádio-ulnar entre cães normais e cães com a doença. Se a IRU é um fator presente na patofisiologia da FPC e doença do compartimento medial do cão, ela pode ser transitória durante 0 crescimento esquelético e parece não estar presente no tempo do diagnóstico da doença. D essa forma 
o uso da osteotomia ou ostectomia da ulna ou rádio para restauração do alinhamento normal da articulação está contraindicado. O utra explicação para tal fato seria que a incongruência pode estar relacionada com apoio do membro e sofrer ação das forças de reação do solo e, quando a imagem tomográfica é obtida, com o animal em decúbito, não há ação dessas forças, não havendo neste momento a incongruência. Como outra limitação desse estudo é que a TC não avalia cartilagem e sim osso subcondral, Kramer et al. (2006) sugerem a obtenção de imagens com utilização de contraste ou a realização de ressonância magnética.

Tomografia computadorizada e ressonância magnética são as técnicas de maior acurácia e sensibilidade para diagnosticar fragmentação do processo coronoide, quando comparadas com a radiografia (HOLSWO RTH et al., 2005).

Snaps et al. (1997) compararam a radiografia, a ressonância magnética (RM) e achados cirúrgicos em cães com displasia do cotovelo. A ressonância magnética foi útil para detecção de fragmentação do processo coronoide ainda não destacado e não mineralizado. As imagens foram consistentemente correlacionadas com os achados cirúrgicos. A RM mostrou-se como uma técnica de alta sensibilidade e acurácia para diagnóstico de fragmentação do processo coronoide, especialmente útil quando os achados radiográficos foram inconclusivos.

Artroscopia pode ser utilizada no diagnóstico de incongruência. A credibilidade da técnica é, no entanto, questionada, uma vez que a introdução do artroscópio no cotovelo pode induzir ou mascarar a presença de incongruência em alguns casos (BEALE; HULSE; SCHULZ, 2003). É uma técnica invasiva e cara, o que limita sua utilidade diagnóstica (SNAPS et al., 1997).

A artrotomia exploratória permite, assim, como a artroscopia, a inspeção direta da superfície articular; o campo visual obtido através da artrotomia é, no entanto, limitado, além de ser um procedimento diagnóstico invasivo (WIND, 1986).

\section{Tratamento}

A incongruência é frequentemente acompanhada de outras lesões do cotovelo como FPC, NUPA e O CD . D essa forma, o tratamento cirúrgico dessa articulação visa não apenas à restauração da congruência articular, mas também remover os fragmentos livres (MORGAN; WIND, 1999).

A presença de incongruência sugere um prognóstico pior após o tratamento cirúrgico da displasia do cotovelo (MORGAN; WIND , 1999), justificando o índice de 30 a $40 \%$ de cães com graus variáveis de dor após o procedimento cirúrgico (READ et al., 1990).

0 acesso cirúrgico do cotovelo vai depender da região a ser abordada, sendo o acesso medial para o processo coronoide medial da ulna e a tróclea do úmero, acesso da porção caudal e proximal da ulna através de incisão linear iniciando-se na tuberosidade do olécrano e acesso do processo ancôneo da ulna pela face lateral da articulação (SCHUL; KROT SCHECK, 2003).

A abordagem das porções caudal e proximal da ulna deve ser realizada com 0 animal em decúbito dorsal com o membro afetado estendido em direção cranial. Realiza-se incisão linear com início na tuberosidade do olécrano até o terço proximal ou médio da ulna, sendo necessária mínima divulsão da fáscia e subcutâneo para acessar a diáfise da ulna. A fascia antebraquial profunda deve ser incisada entre o flexor e o extensor do carpo para permitir a retração lateral e medial da musculatura. D issecam-se as porções da musculatura da face cranial da ulna e as mantêm retraídas (SCHULZ; KROTSCHECK, 2003).

0 acesso à tróclea umeral e ao processo coronoide medial da ulna é realizado com 0 animal em decúbito dorsal, com o membro afetado, tracionado lateralmente e utilizando-se a extremidade da mesa como fulcrum com intuito de expor, de forma mais efetiva, a porção medial da articulação. Realizase incisão curva da pele pela face medial da articulação na altura do epicôndilo medial do úmero estendendo-se até o terço proximal da ulna. D eve-se então realizar incisão da fascia antebraquial e exposição do músculo pronador e flexor carpo radial com posterior divulsão entre esses dois músculos, sendo estes mantidos afastados (SCHULZ; KROTSCHECK, 2003).

Já o processo ancôneo é acessado pela face lateral da articulação, uma vez que o epicôndilo lateral do úmero apresenta menores proporções que o medial, propiciando uma melhor exposição e 
remoção do fragmento livre. Com o animal em decúbito lateral, de forma que o membro afetado seja 0 de cima, realiza-se incisão curva da pele estendendo-se do epicôndilo lateral do úmero até o início da cabeça do radio, sendo a incisão da fascia do subcutâneo realizada na mesma orientação que a pele, expondo a porção cranial da fascia do músculo tríceps, a qual deve ser incisada imediatamente da cranial ao músculo tríceps, sendo este retraído caudalmente expondo o músculo ancôneo. 0 músculo ancôneo e a cápsula subjacente devem ser incisados longitudinalmente através do centro do músculo e mantidos retraídos para permitir a exposição do processo ancôneo (SCHULZ; KROTSCHECK, 2003).

A osteotomia da ulna é o procedimento mais frequentemente realizado na correção da incongruência e amplamente aceito para o tratamento da NUPA como alternativa para a remoção cirúrgica do fragmento livre (MEYER-LINDENBERG et al., 2001). 0 procedimento gera uma melhor distribuição da pressão na articulação do cotovelo (PRESTON et al., 2000). A orientação da osteotomia pode ser perpendicular ou oblíqua ao eixo longitudinal da ulna, sendo que a osteotomia oblíqua no sentido caudo-proximal para crânio-distal evita a acomodação cranial e proximal extremas, diminui a mobilidade da porção proximal da ulna, evitando, dessa forma, a formação de calo ósseo exuberante e retardo da consolidação (NESS, 1998). A fusão do processo ancôneo nem sempre ocorre após a osteotomia da ulna, alguns autores recomendando a adição de parafuso de compressão para a fixação do fragmento (SCHULZ; KROTSCHECK, 2003).

A primeira descrição da técnica com osteotomia oblíqua foi realizada por Thomson et al. (1995) em três cotovelos com NUPA obtendo êxito no tratamento em duas articulações. Sugere-se tratamento de tal afecção por osteoto mia da ulna por proporcionar uma diminuição na pressão no ponto de contato da tróclea do úmero e o processo ancôneo, permitindo, dessa forma, a união óssea.

Já Sjöström, Kasstron e Kallberg (1995) executaram a osteotomia perpendicular em 22 cotovelos de cães com NUPA, obtendo 70\% dos animais com retorno total à função do membro, sendo que os 30\% restantes apresentaram dor residual, a qual pôde ser atribuída a diferentes fatores, como artrose, consolidação precoce da osteotomia da ulna e conformação anormal da tróclea.

A osteotomia da ulna também é descrita como procedimento adicional no tratamento cirúrgico da FPC. Em estudo com 10 cães, todos com menos de 10 meses, foi realizada a osteotomia e a retirada do fragmento por artrotomia, resultando em melhora importante em 9 dos 10 cães com até dois meses de pós-operatório (NESS, 1998).

Em estudo com 83 cães adultos com FPC submetidos à remoção do fragmento por artroscopia e associação da osteotomia da ulna a taxa de sucesso foi de 93\%, apesar do desenvolvimento progressivo de osteoartrose (BARDET; BUREAU, 1996).

Para incongruência discreta do cotovelo, resultante de encurtamento do rádio, também é descrita a técnica de osteotomia de encurtamento ulnar, a qual promoverá uma diminuição no comprimento daulna, permitindo uma melhor congruência da articulação. No entanto, se tal encurtamento for acarretar em prejuízo ao animal devido à redução do tamanho do membro, a técnica mais recomendada é a de osteotomia de prolongamento do rádio (HULSE; JO HNSO N, 2002).

As variações da técnica de osteotomia da ulna bem como seus resultados parecem ser bem variáveis. Alguns autores relatam a necessidade de uma osteotomia proximal e oblíqua combinada com pino intramedular para evitar formação de deformidade do tipo varus e interferência do ligamento interósseo na recuperação da congruência articular (PRESTO N et al., 2000), enquanto outros autores referem que a ausência da utilização do pino intramedular não resultou em deformidade do tipo varus (BARDET et al., 1996) e que a osteotomia distal da ulna em cães com até nove meses de idade acometidos por FPC e incongruência articular gerou bons resultados em 77,5\% dos pacientes. Como principal complicação da técnica é relatada a quebra do pino intramedular (BARDET et al., 1996), podendo ocorrer também sua migração.

O alongamento radial é uma técnica desenvolvida mais recentemente com o objetivo de reduzir o "degrau" entre rádio e ulna gerado pela incongruência (SLOCUM; PFEIL, 2004). Um estudo realizado em cinco cães, apresentando encurtamento do rádio induzido iatrogenicamente, obteve retorno da congruência normal da articulação do cotovelo em todos os animais, porém foram observadas a recorrência da incongruência e claudicação em três dos animais (OLSSO N; CARRIG ; BRINKER, 1981).

Rev. Acad., Ciênc. Agrár. Ambient., Curitiba, v. 6, n. 3, p. 413-423, jul./ set. 2008 
Em um relato de caso de Altunatmaz et al. (2003), sobre tratamento por osteotomia do rádio, associado à estabilização com o uso de fixador externo de Ilizarov na incongruência do cotovelo, devido ao fechamento precoce do disco epifisário distal do rádio em um Pastor Alemão, Altunatmaz et al. (2003) mostram resultado satisfatório, observando ausência de dor no membro e bom retorno de sua função, porém ressaltam a necessidade de mais estudos clínicos a longo prazo, bem como as possíveis complicações da técnica.

O utras técnicas pouco difundidas e com escassez de estudos como a osteotomia lateral ou medial do úmero, a qual é considerada como uma técnica que não soluciona as alterações de pressão e o padrão de contato entre as superfícies articulares do cotovelo (FUJITA et al., 2003), e a coronoidectomia, técnica descrita por Puccio et al. (2003) para tratamento de cães com FPC em que se realiza artrotomia e osteotomia de $4 \mathrm{~mm}$ ou mais do processo coronoide medial, também são descritas.

O tratamento artroscópico apresenta-se como uma boa opção, quando a incongruência do cotovelo apresenta-se concomitantemente com as demais afecções da displasia do cotovelo.

As afecções do cotovelo mais comuns e passíveis de tratamento por artroscopia são FPCM, OCD e a NUPA. O exame artroscópico de tais afecções frequentemente revela lesões teciduais mais extensas do que as observadas ao exame radiográfico.

0 estabelecimento dos portais na face medial da articulação do cotovelo permite inspeção quase completa do espaço articular, bem como o tratamento das lesões em processo ancôneo, processo coronoide medial e côndilo umeral medial (SCHULZ; KROTSCHECK, 2003).

Samoy et al. (2006) realizam estudo através de inspeção artroscópica do cotovelo e remoção ampla do processo coronoide em cães com incongruência severa do cotovelo e obtiveram, até o presente momento, bons resultados com relação ao retorno da função do membro e alívio da dor, embora a artrose, inflamação e lesões em cartilagem já se apresentavam estabelecidas.

\section{CONSIDERAÇÕES FINAIS}

Através dos trabalhos consultados para a presente revisão sobre a incongruência do cotovelo, observa-se forte relação entre ela e as demais afecções do cotovelo, porém, a discussão sobre a sua inclusão na displasia do cotovelo e seu papel como etiologia das demais afecções permanecerá até que futuros estudos demonstrem o real papel da incongruência como fator predisponente ou como quarta afecção da DC.

O diagnóstico radiográfico da incongruência é complexo, pois é muito comum a ocorrência de sobreposição de imagens, seu posicionamento é difícil e há necessidade de um radiologista experiente para a realização do procedimento. Porém, é um método muito importante e amplamente utilizado para triagem e possui maior disponibilidade para o médico veterinário.

A TC e a RM são as técnicas de diagnóstico de maior acurácia e de maior sensibilidade para o diagnóstico de FPC. A TC também tem sido uma ferramenta importante na detecção de incongruência do cotovelo, sendo, atualmente, o melhor método de diagnóstico da IC. A RM é pouco viável por seu alto custo e indisponibilidade, porém tem um grande potencial diagnóstico, necessitando de pesquisas futuras.

O diagnóstico deveserprecoceparaqueacorreção cirúrgicatenhamelhorprognóstico, principalmente antes do aparecimento da degeneração osteoarticular secundária e do crescimento completo do animal. 0 prognóstico dos animais com incongruência é o pior dentre as afecções que compõem a displasia do cotovelo.

0 tratamento visa não somente à retirada de fragmentos articulares livres, mas também a correção da incongruência, uma vez que a presença desta está diretamente relacionada a um prognóstico pobre no pós-operatório. A osteotomia proximal oblíqua da ulna com estabilização da porção proximal da ulna com pino intramedular é o procedimento mais utilizado para o tratamento da incongruência, sendo indicado preferencialmente antes do fechamento da linha de crescimento, observando-se bons resultados quanto ao retorno da função do membro.

Há um aumento constante das pesquisas relacionadas à incongruência do cotovelo, envolvendo sua fisiopatogenia, formas de diagnóstico e tratamento, na tentativa de um maior entendimento desta afecção, para que se evite e diminua a progressão da degeneração osteoarticular secundária. 


\section{REFERÊNCIAS}

ALTUNATMAZ, $\mathrm{K}$. et al. Treatment by the use of an Ilizarov external fixator of incongruity in the elbow joint due to premature closure of the distal radial growth plate in a German Shepherd. Turkish Journal of Veterinary and Animal Sciences, v. 27, p. 1235-1239, 2003.

BARDET, J. F.; BUREAU, S. La fragmentation du processus coronoïde chez le chien. Pratique Medicale et Chirurgicale de L'Animal de Compagnie, v. 31, p. 451-463, 1996.

BEALE, B. S.; HULSE, D. A.; SCHULZ, K. S. Arthroscopically assisted surgery of the elbow joint. In: Small Animal Arthroscopy. Philadelphia, PA: Saunders, 2003. p. 51-79.

BLO ND, L. et al. Sensitivity and specificity of radiographic detection of canine elbow incongruence in an in vitro model. Veterinary Radiology and Ultrasound., v. 46, n. 3, p. 210-216, 2005.

BOULAY, J. P. Fragmentation medial coronoid process of th ulna in the dog. The Veterinary clinics of North America, v. 28, n. 1, p. 51-75, 1998.

DYCE, K. M.; SACK, W. O.; WENSING, C. J. G. Tratado de anatomia veterinária. 3. ed. São Paulo: Elsevier, 2004.

FUJITA, Y. et al. Effect of humeral osteotomy on joint surface contact in canine elbow joints. Am. J. Vet. Res., v. 64, 4, p. 506-511, 2003.

GEMMILL, T. J. et al. Use of reconstructed computed tomography for the assessment of joint spaces in the canine elbow. American Journal of Veterinary Research., v. 47, p. 66-74, 2006.

HOLSWORTH, I. G. et al. Accuracy of computerized tomographic evaluation of canine radioulnar incongruence in vitro. Veterinary Surgery., v. 34, 2, p. 108-113, 2005.

HORNOF, W. J. et al. Canine elbow dysplasia. The early radiographic detection of fragmentation of the coronoid process. American Journal of Veterinary Research., v. 30, p. 257-266, 2000.

HULSE, D. A.; JOHNSO N, A. L. Tratamento da doença articular. In: FO SSUM, T. W. Cirurgia de pequenos animais. São Paulo: Roca, 2002. p. 978-1103.

KIRBERGER, R. M.; FOURIE, S. L. Elbow dysplasia in the dog: pathophysiology, diagnosis and control. Journal of South Africa Veterinary Association, v. 69, n. 2, p. 43-54, 1998.

KRAMER, A. et al. Computed Tomographic evaluation of canine radioulnar incongruence in vivo. Veterinary Surgery., v. 35, p. 24-29, 2006.

MASO N, D. R.; SCHULZ, K. S.; SAMII, V. F. Sensitivity of radiographic evaluation of radioulnar incongruence en the dog in vitro. Veterinary Surgery., v. 31, n. 2, p. 125-132, 2002.

MEYER-LINDENBERG, A. et al. Short and long-term results after surgical treatment of an ununited anconeal process in the dog. Veterinary and Comparative Orthopaedics Traumatology, v. 14, p. 101-110, 2001.

MORGAN, J. P.; WIND, A. O steochondroses, hip displasia, elbow displasia. In: MORGAN, J. P. WIND A, D AVID SO N, A. Hereditary bone and joint diseases in the dog. Hanover: Schlütersche GmbH \& Co., 1999. p. 41-94.

MURPHY, S. T. et al. Effect of radiographic positioning on interpretation of cubital joint congruity in dogs. American Journal of Veteninary Research., v. 59, n. 11, p. 1351-1357, 1998.

NESS, M. G. Treatment of fragmented coronoid process in young dogs by proximal ulnar osteotomy. Journal Small Animal Practice, v. 39, p. 15-18, 1998. 
OLSSO N, N. C.; CARRIG, C. B.; BRINKER, W. O. Asynchronous growth of the canine radius and ulna: surgical correction following experimental premature closure of the distal radial physis. Veterinary Surgery., v. 10, p. 125-131, 1981.

OLSSON, S. E. The early diagnosis of fragmented coronoid process and osteochondrosis dissecans of the canine elbow joint. Journal of the American Hospital Association., v. 19, n. 6, p. 616-626, 1983.

PRESTON, C. A.; SCHULZ, K. S.; KASS, P. H. InVitrodetermination of contact areas in the normal elbow joint of dogs. American Journal Veterinary Research, v. 61, n. 10, p. 1315-1321, 2000.

PRESTON, C. A. et al. In Vitro experimental study of the effect of radial shortening and ulnar ostectomy on contact patterns in the elbow joint of dogs. American Journal Veterinary Research, v. 62 , n. 10, p. 1548-1556, 2001.

PUCCIO, M. et al. Clinical evolution and long-term follow-up of dogs having coronoidectomy for elbow incongruity. Journal of the American H ospital Association., v. 39, p. 473-478, 2003.

READ, R. A. et al. Fragmentation of the medial coronoid process of the ulna in dogs: a study of 109 cases. Journal Small Animal Practice, v. 31, p. 330-334, 1990.

REICHLE, L. K.; PARK, R. D.; BAHR, A. M. Computed tomography findings of dogs with cubital joint lameness. Veterinary Radiology and Ultrassound, v. 41, p. 125-130, 2000.

REMY, D. et al. Canine elbow displasia and primary lesions in german shepherd dogs in France. Journal Small Animal Practice, v. 45, p. 244-248, 2004.

ROVESTI, G. L. et al. The use of computed tomography in the diagnostic protocol of the elbow in the dog: 24 joints. Veterinary Comparative Orthopedies and Traumatology, v. 15, p. 35-43, 2002.

SAMOY, Y. et al. Elbow Incongruity in the Dog. Veterinary Comparative Orthopedies and Traumatology, v. 19, p. 1-8, 2006.

SCHWARZ, P. D. Canine elbow dysplasia. In: BONAG URA, J. D. Kirk's current veterinary therapy. 13. ed. Philadelphia: WB Saunders, 2000. p. 1004-1014.

SJÖ STRO M, L.; KASSTRON, H.; KALLBERG, M. Ununited anconeal process in the dog. Pathogenesis and treatment by osteotomy of the ulna. Veterinary Comparative Orthopedies and Traumatology, v. 8, p. 170-176, 1995.

SCHULZ, K. S.; KROTSCHECK, U. Canine elbow displasia. In: SLATTER, D. Textbook of small animal surgery. Philadelphia: Saunders, 2003. p. 1927-1952.

SLOCUM, B.; PFEIL, I. Radius elongations for pressure relief of the coronoid process of the ulna. In: ESVOT CONGRESS, 12., 2004. Munich. Proceedings... Munich, 2004.

SNAPS, F. et al. Comparison of radiography, magnetic resonance imaging, and surgical findings in dogs with hip dysplasia. American Journal of Veterinary Research., v. 58, p. 1367-1370, 1997.

THOMPSO N, M. J.; ROBBINS, G. M. O steochondrosis of the elbow: a review of the pathogenesis and a new approach to treatment. Australian Veterinary Journal., v. 72, p. 375-378, 1995.

WIND, A. P. Elbow incongruity and developmental elbow diseases in the dog: Part I. Journal of the American Hospital Association., v. 22, n. 6, p. 711-724, 1986.

Recebido: 05/ 05/2008

Recived: 05/ 05/ 2008

Aprovado: $14 / 05 / 2008$

Approved: 05/ 14/ 2008 